\title{
Urban security and crime prevention in smart cities: a systematic review
}

\author{
Gerard Vivo-Delgado ${ }^{\mathrm{a}}$, Francisco J. Castro-Toledo ${ }^{\mathrm{bc}}$ \\ ${ }^{a}$ PhD student, Universidad Miguel Hernández de Elche \\ ${ }^{\mathrm{b}}$ Centro CRÍMINA para el estudio y prevención de la delincuencia, Universidad Miguel Hernández \\ de Elche \\ ${ }^{\mathrm{c}}$ Plus Ethics
}

\begin{abstract}
The scientific literature on crime prevention in urban contexts is very vast, nevertheless it is still limited in the Smart Cities context. With the aim of assessing how this phenomenon has been studied to date from an evidence-based approach, a quantitative systematic review has been conducted on the basis of gathering information from the most recent scientific literature (20142019) on strategies for improving urban safety in smart city scenarios $(n=20)$. Specifically, it was systematised according to different variables such as research designs, results of the implementation of measures to improve urban safety, crimes assessed, fields of application of the measures and problems associated with the use of these measures. As a result, the majority of the studies examined are of a cross-sectional, non-experimental nature. Also, most results are descriptive and the preventive measures evaluated have positive effects. Similarly, most of the applications are connected with civil, preventive and police settings. Finally, this paper analyses the importance of following up on this emerging line of research.
\end{abstract}

Keywords: smart cities, crime prevention, smart policing, urban security, systematic review.

\footnotetext{
${ }^{1}$ Corresponding author: gerarddvd@gmail.com
} 


\section{Theoretical framework}

As the population grows in cities and the scarcity of resources increases (Townsend, 2013; Eremia, Toma \& Sanduleac, 2017), it is changing the way cities are being managed to be more efficient and, therefore, smarter in terms of the application of new technologies and the use of the available resources (Maestre, 2015; Kummitha, 2019). Such conventional cities are moving towards the so-called "smart cities" (Kummitha, 2019). Broadly speaking, a Smart City is defined by the ability to create or adopt solutions to the challenges and opportunities of transforming cities into more productive and liveable places for their citizens (Alawadhi et al., 2012; Vidal-Tejedor, 2015; Mueller, 2017), by taking advantage of new technologies and the hyper-connectivity of the Internet of Things (Zanella \& Vangelista, 2014).

Among its different fields, security management in smart cities holds a relevant position due to its features and functionalities (Colado, Gutiérrez, Vives \& Valencia, 2014). The smart economy, smart people, smart governance, smart mobility, smart environment and smart life are examples of this (Deloitte, 2015; Khatoun \& Zeadalli, 2016). Vidal-Tejedor (2015) provides several examples as the following. Regarding the Smart economy, the security related to the financial field, such as online fraud, can be identified. Smart people can be defined as services to increase the comfort and security of citizens (alarms, security systems, flood prevention, prevention of social isolation of the elderly, etc.). Smart governance refers to the security of public administration and the use of data systems such as open government data (Lodato, French \& Clark, 2018). Smart mobility covers the monitoring of traffic flows, the detection of driving offences, information on accidents, the prioritisation of emergency vehicles, etc. Under Smart environmental we may find domotics, which includes, for example, prevention of fire or theft. Finally, Smart living 
refers to risk assessment in buildings, town planning and urban sensors, among other examples. Smart cities security would consist, in broad terms, in the organization of disorderly urbanizations, in safer cities through the implementation of hyperconnected sensor networks and security systems, the management of accidents, breakdowns or critical situations. through police, medical and logistics coordination of rescue (among other actors) or computer security and protection of large masses of data (Colado, Gutiérrez, Vives \& Valencia, 2014).

Within this new scope, the most relevant elements for the management of public safety in smart cities are data and its analysis: history, records, road traffic, beliefs, correlations between variables, among other data that may be of interest (Ghosh, Chun, Shafiq \& Adam, 2016). The use of statistical data and its controls can be proposed by Truntsevsky, Lukiny, Sumachev \& Kopytova (2018) as an example through a mobile app that uses geographic information systems that shows the crime levels in an area on a colour map which can be modified based on historical data. The users with the application are able to know the position and modify their route or transport according to their preferences, and the security authorities are able to adapt their support to the preventive requirements of the moment.

When having a large amount of data, Smart cities must consider, precisely, the protection of that data against possible cyberattacks to the city systems (Rivero, 2017). For this, it is of vital importance the encryption of the data (Khatoun \& Zeadally, 2016), the continuous monitoring of the sensors, the performance of audits to the companies that have access to the data, etc. 
Therefore, governments must opt for a balance between transparency and confidentiality at the level deemed convenient (Macmanus, Caruson \& Mcphee, 2013). Citizens must be aware of what data is collected, the manner in which personal data is obtained and the protection given to the personal data to prevent it from a third party or for purposes unrelated to the declared ones (see European General Data Protection Regulation, GDPR).

Likewise, by means of a smartphone you may also have apps that benefit from the data collected in the city and may facilitate the daily routine of the citizen, such as applications that show on a map the most and least lighted streets of a given route (Kumar, 2019). Such an application may be interesting both for citizens, who can change their route on more illuminated streets, and for the city managers, who are able to get an idea of the areas where they have to improve the level of illumination. Furthermore, for emergency management there is, for instance, Bartoli and others' proposal (2013) for a platform that uses ICT and methods of analysis of the whole flow of information that is overlooked in an emergency scenario (ie. first responses, authorities and citizens). Also, they may propose initiatives to foresee possible terrorist acts by using data from different intelligence sources together with algorithms, as is done in law enforcement agencies (Sormani et al., 2016). Lastly, the use of drones is also becoming more common in rescue operations due to their size, their ease of movement and high-definition cameras carried that make possible to monitor the area in which they are located (Vattapparamban et al., 2016).

In view of the range of choices available in smart cities, it is necessary to know what and how- has been done in relation to security in this context previously. This will enable 
us to summarize relevant information that will help us to understand the state of the art and to identify shortcomings or strengths to be addressed. This study is intended to be a means of gathering previous evidence and setting new benchmarks for the scientific community.

This study is organized into the following sections: the objectives of the study; a methodological section which describes the approach used to conduct the quantitative systematic review, including the steps followed and the variables analyzed; a results section, both at qualitative and quantitative terms; a conclusion section and a discussion section.

\section{The present study}

Considering that nowadays there are very few studies on crime prevention in smart cities and cities are growing very quickly through many technological achievements which can be used to this end, the main objective of this systematic review is to assess how security in smart cities has been studied to date from an evidence-based approach. To find out, several specific objectives are proposed, including the following:

1. To identify the more common features of the studies, including which countries are leading in the publication of research in this field and the disciplines of the journals in which they have been published;

2. To delimit the common designs of the studies that were conducted;

3. To better understand the efficacy of the measurements taken in the research;

4. To specify the level of interest that has been raised by the implementation of artificial intelligence in related projects;

5. To identify the criminal behaviours most often targeted for prevention; 
6. To set out the principal fields of implementation of the used measures; and

7. To detect challenges associated with the performance of the research.

\section{Data and methodology}

\section{a. Systematic Quantitative Literature Review}

A quantitative systematic review of literature was conducted for this study in line with PRISMA guidelines (Moher, Liberati, Tetzlaff \& Altman, 2009) and Pickering \& Byrne (2014) in order to identify peer-reviewed papers on crime prevention strategies in smart cities.

These reviews consist of a scientific literature query using online databases and other sources to find the major published studies of a given topic. With these studies, the expert builds his/her own database with the appropriate information to assess the state of the art of the literature in the fields. Such a method is recommended for reliable, quantifiable and reproducible findings, and for identifying gaps in the field (Pickering \& Byrne, 2014).

\section{b. Literature search}

Both Scopus and ProQuest were used as databases. They were chosen because of their broad range of papers and their high reputation as evidence-based search engines. In the beginning, the keywords to be searched in the databases were carefully chosen based on the topic of study. As a result, keywords such as Intelligent Cities, Crime Prevention, Artificial Intelligence, Criminology, Intelligent Technology, Criminal Intelligence, Security, Intelligent Police, among other examples, yielded very poor results. So finally, the selected keywords were "crime prevention" OR "security" AND "smart cities" OR "artificial intelligence". 
Furthermore, studies were limited to full text articles (not only abstracts or summary of papers), peer-reviewed, published in scientific journals in English and Spanish, in the last 5 years (to restrict the sample to only current papers) and the kind of contribution (article or principal article).

\section{c. Selection of studies}

The study selection process was organized in six stages to be incorporated in the quantitative systematic review. Those stages are outlined in the following flowchart (see Figure 1):

1. The identification of the studies on specialised scientific databases.

2. A record after the filters are applied: studies identified after applying the filters indicated above.

3. Identification of titles duplicated: filtering by the headings duplicated from both databases and remove them.

4. Abstract screening: scanning the abstracts of the papers to find out whether the paper is about crime prevention in smart cities.

5. Full article review: full text reading to gain in-depth knowledge of the content and ultimately to select it for consideration.

6. Studies included in the quantitative systematic review 
Figure 1. Flow Diagram of the systematic review

1.Records identified through database searching

- $(\mathrm{n}=11.368)$

2. Records after apply filters

- $(\mathrm{n}=189)$

3. Records after duplicates removed

- $(\mathrm{n}=91)$

4. Records screened by abstract

- $(n=58)$

5. Full text articles assessed for eligibility

- $(n=33)$

\section{Studies included in quantitative review$$
\text { - }(n=20)
$$

\section{d. Selected variables}

Variables are organized by categories according to the features to be compared (see Table 1). In order to respond to the objectives listed above, the following variables have been chosen.

Under the first category, the features of the study such as location, year of publication and journal discipline are considered, because they are basic variables for finding out more about the state of academic interest. The following category is focused on the research design: timing of data collection, approach and research method, to understand whether 
these studies have been conducted at specific points in time or have continued over time, whether they have available data that can be compared quantitatively at different points in time or have been based on qualitative data, or whether they have conducted entirely experimental studies or have chosen less experimental options. Other category looks at the results through statistics (descriptive or inferential) and the effects of the obtained result from being aware of the results and identifying which measures have been effective and which have not, as well as finding out whether the results are descriptive or may have been taken further by means of inferential statistics. The security strategy behind each study is also categorized (whether they use Artificial Intelligence or unsystematized strategies), as well as the typology of the criminal behavior to be prevented and the implementation of the preventive measures given that understanding the issues to be faced is central and will determine the measures to be implemented, as will understanding the use or lack thereof of artificial intelligence systems. There is finally a category on the challenges and limitations faced in the studies through the difficulties that other researchers experienced, it is possible to explore alternatives or improvements without dragging out the previous challenges for future research. 
Table 1. Categories and variables of the study.

\begin{tabular}{|c|c|c|}
\hline Category & Variables & Modalities \\
\hline \multirow{7}{*}{ Study characteristics } & Location & Country \\
\hline & & Law \\
\hline & & Computing / Technology \\
\hline & Journal discipline & Security \\
\hline & & Criminology \\
\hline & & Sociology / Political science \\
\hline & & Ethics \\
\hline \multirow{8}{*}{ Design } & Data collection timino & Transversal \\
\hline & Data contection timing & Longitudinal \\
\hline & & Quantitative \\
\hline & Approach & Qualitative \\
\hline & & Mixed \\
\hline & & Experimental \\
\hline & Research method & Quasi-experimental \\
\hline & & Non-experimental \\
\hline \multirow{7}{*}{ Results } & & Descriptive \\
\hline & Statistics scope & Inferential \\
\hline & & Mixed \\
\hline & & Positive \\
\hline & Effect of the result obtained & Negative \\
\hline & & Mixed \\
\hline & & Null \\
\hline & & Use Artificial Intelligence \\
\hline Security strategy & Measures used & Use conventional strategies \\
\hline \multirow{7}{*}{ Crime prevention } & & Incivism/vandalism \\
\hline & & Against collective security \\
\hline & & Against sexual indemnity \\
\hline & Crime typology & Against heritage \\
\hline & & Homicide \\
\hline & & Injuries \\
\hline & & Other crimes \\
\hline \multirow{9}{*}{ Application } & & Civil \\
\hline & Use & Military \\
\hline & & Dual \\
\hline & & Investigation \\
\hline & Objective & Mitigation \\
\hline & & Prevention \\
\hline & & Police \\
\hline & Field & Judicial \\
\hline & & Both \\
\hline \multirow{4}{*}{ Limitations } & \multirow{4}{*}{ Difficulties associated } & Data management \\
\hline & & Decision making \\
\hline & & Effective implementation \\
\hline & & Other problems \\
\hline
\end{tabular}

Note. The encoding was designed to synthesize the information of each paper and to ease its review. The number "1" was allocated for those modalities of the variable observed in the article, "0" for the modalities that were not given, and "99" for the modalities that were not specified. 


\section{Results}

\section{a. Study characteristics}

Concerning the first objective of the review about the more common features of the studies, findings show that about half of the studies were conducted in Europe $(40 \%$ of them in the UK) and 35\% in Asia, of which 57\% came from South Korea, placing South Korea at the forefront of research production in the field. Likewise, most of the papers were published in security journals $(25 \%)$ or in sociology and political science journals (25\%), being followed by technological journals $(22 \%)$.

As regards the design of the studies, three aspects are of interest. The first is the timing of data collection, with $70 \%$ of the data being of a cross-sectional nature. Secondly, that most studies take a qualitative or mixed approach (90\%), while only two studies are quantitative. Lastly, the used research method are mainly non-experimental or quasiexperimental studies (95\%), with only one study being experimental. These results are probably due, in part, the difficulty of conducting longitudinal studies, even more so on such current issues, as these have been under development for a short time, as well as the difficulty of considering such experimental studies in the context of smart cities.

\section{b. Effectiveness of the measures}

The findings obtained by the different papers are mostly descriptive (90\%) with positive effects in $60 \%$ as compared to a unique study that obtained negative results $(5 \%)$. Furthermore, up to $30 \%$ of the studies obtained both positive and negative effects (i.e. mixed effect). Given these data, the measures implemented in the different studies were found to be effective. 


\section{c. Use of Artificial Intelligence}

Use of AI potentially opens up a wide range of avenues for enhancing urban safety in cities. However, only about half of the studies reviewed have used AI in their research, while the other half have used conventional or unsystematized approaches. AI strategies addressed in the studies are largely in terms of data analysis $(75 \%)$ and the use of multimedia systems (45\%), as might be the case of CCTV.

\section{d. Application of the measures}

Most of the crimes studied are social and physical crimes (69.3\%), which include vandalism/incivism, community security and property crimes. Whereas the remaining crimes are more against the person (30.7\%), such as homicide, injuries and/or sexual crimes. Furthermore, the use of its application has been almost entirely civil (95\%), for the purpose of preventing crime $(74 \%)$ or mitigating it $(21.7 \%)$, and conducted from police settings $(100 \%)$

\section{e. Difficulties associated}

Lastly, concerning the last target of this quantitative systematic review, researchers met with challenges during their studies. These challenges are mainly associated with data management (44.4\%) and the effective implementation of their approaches (26\%), as well as with decision-making issues (25\%). Additionally, several papers identified poor access to data on criminal activities in their countries and the absence of any standards or guidelines that could be followed on crime prevention in smart cities given the scarce information available. 


\section{f. Qualitative systematic review of literature}

Table 2. Summary of qualitative results of the systematic review

\begin{tabular}{|c|c|c|c|c|c|c|c|}
\hline Authors, year and country & Discipline & Design & Results & Security strategy & Crime typology & Area of application & Limitations \\
\hline $\begin{array}{l}\text { Elmaghraby, A. S. } \\
\text { Losavio, M. M. } \\
\text { (2014, United States) }\end{array}$ & $\begin{array}{l}\text { Security } \\
\text { Ethics }\end{array}$ & $\begin{array}{l}\text { Transversal } \\
\text { Qualitative } \\
\text { Non-experimental }\end{array}$ & $\begin{array}{l}\text { Descriptive } \\
\text { Mixed effect }\end{array}$ & $\begin{array}{l}\text { Use IA } \\
\text { Data analysis }\end{array}$ & Not specified & $\begin{array}{l}\text { Civil use } \\
\text { Crime mitigation } \\
\text { Police field }\end{array}$ & $\begin{array}{l}\text { Data management } \\
\text { Effective implementation }\end{array}$ \\
\hline $\begin{array}{l}\text { Byun, J. Y. } \\
\text { Nasridinov, A. } \\
\text { Park, Y. H. } \\
\text { (2014, South Korea) }\end{array}$ & $\begin{array}{l}\text { Computing } \\
\text { Technology }\end{array}$ & $\begin{array}{l}\text { Transversal } \\
\text { Qualitative } \\
\text { Quasi-experimental }\end{array}$ & $\begin{array}{l}\text { Descriptive } \\
\text { Positive effect }\end{array}$ & $\begin{array}{l}\text { Use IA } \\
\text { Data analysis } \\
\text { Multimedia systems }\end{array}$ & Not specified & $\begin{array}{l}\text { Civil use } \\
\text { Crime mitigation } \\
\text { Police field }\end{array}$ & Not specified \\
\hline $\begin{array}{l}\text { Carreño, P. } \\
\text { Gutierrez, F. J. } \\
\text { Ochoa, S. F. } \\
\text { Fortino, G. } \\
\text { (2015, Chile) }\end{array}$ & $\begin{array}{l}\text { Computing } \\
\text { Technology } \\
\text { Sociology } \\
\text { Political science }\end{array}$ & $\begin{array}{l}\text { Transversal } \\
\text { Mixed } \\
\text { Quasi-experimental }\end{array}$ & $\begin{array}{l}\text { Descriptive } \\
\text { Mixed effect }\end{array}$ & $\begin{array}{l}\text { Use conventional strategies } \\
\text { Multimedia systems }\end{array}$ & $\begin{array}{l}\text { Incivism/Vandalism } \\
\text { Crimes against heritage and against } \\
\text { the socio-economic order }\end{array}$ & $\begin{array}{l}\text { Civil use } \\
\text { Crime prevention } \\
\text { Police field }\end{array}$ & Data management \\
\hline $\begin{array}{l}\text { Chiodi, S. I } \\
\text { (2016, Italy) }\end{array}$ & Criminology & $\begin{array}{l}\text { Transversal } \\
\text { Qualitative } \\
\text { Non-experimental }\end{array}$ & $\begin{array}{l}\text { Descriptive } \\
\text { Mixed effects }\end{array}$ & $\begin{array}{l}\text { Use conventional strategies } \\
\text { CPTED } \\
\text { Citizen participation }\end{array}$ & Incivism/Vandalism & $\begin{array}{l}\text { Civil use } \\
\text { Crime prevention } \\
\text { Police field }\end{array}$ & Effective implementation \\
\hline $\begin{array}{l}\text { Arikuma, T. } \\
\text { Mochizuki, Y. } \\
\text { (2016, Japan) }\end{array}$ & Other & $\begin{array}{l}\text { Longitudinal } \\
\text { Qualitative } \\
\text { Quasi-experimental }\end{array}$ & $\begin{array}{l}\text { Descriptive } \\
\text { Positive effect }\end{array}$ & $\begin{array}{l}\text { Use IA } \\
\text { Multimedia systems }\end{array}$ & Other crimes & $\begin{array}{l}\text { Civil use } \\
\text { Crime prevention } \\
\text { Police field }\end{array}$ & Data management \\
\hline $\begin{array}{l}\text { Lin, Y. L. } \\
\text { Chen, T. Y. } \\
\text { Yu, L. C. } \\
\text { (2017, Taiwan) }\end{array}$ & $\begin{array}{l}\text { Computing } \\
\text { Technology }\end{array}$ & $\begin{array}{l}\text { Transversal } \\
\text { Quantitative } \\
\text { Quasi-experimental }\end{array}$ & $\begin{array}{l}\text { Inferential } \\
\text { Positive effect }\end{array}$ & $\begin{array}{l}\text { Use IA } \\
\text { Data analysis }\end{array}$ & $\begin{array}{l}\text { Incivism/Vandalism } \\
\text { Crimes against collective security }\end{array}$ & $\begin{array}{l}\text { Civil use } \\
\text { Crime prevention } \\
\text { Police field }\end{array}$ & Not specified \\
\hline $\begin{array}{l}\text { Perrot, P. } \\
\text { (2017, France) }\end{array}$ & $\begin{array}{l}\text { Security } \\
\text { Criminology } \\
\text { Sociology } \\
\text { Political science } \\
\end{array}$ & $\begin{array}{l}\text { Longitudinal } \\
\text { Mixed } \\
\text { Quasi-experimental }\end{array}$ & $\begin{array}{l}\text { Descriptive } \\
\text { Positive effect }\end{array}$ & $\begin{array}{l}\text { Use IA } \\
\text { Data analysis }\end{array}$ & $\begin{array}{l}\text { Crimes against heritage and against } \\
\text { the socio-economic order }\end{array}$ & $\begin{array}{l}\text { Civil use } \\
\text { Crime prevention } \\
\text { Police field }\end{array}$ & $\begin{array}{l}\text { Data management } \\
\text { Decision making }\end{array}$ \\
\hline $\begin{array}{l}\text { Prislan, K. } \\
\text { Slak, B. } \\
\text { (2018, Slovenia) }\end{array}$ & $\begin{array}{l}\text { Law } \\
\text { Security } \\
\text { Criminology }\end{array}$ & $\begin{array}{l}\text { Transversal } \\
\text { Qualitative } \\
\text { Non-experimental }\end{array}$ & $\begin{array}{l}\text { Descriptive } \\
\text { Positive effect }\end{array}$ & $\begin{array}{l}\text { Use IA } \\
\text { Data analysis } \\
\text { Multimedia systems }\end{array}$ & Not specified & $\begin{array}{l}\text { Civil use } \\
\text { Crime prevention } \\
\text { Crime mitigation } \\
\text { Police field }\end{array}$ & $\begin{array}{l}\text { Data management } \\
\text { Effective implementation }\end{array}$ \\
\hline $\begin{array}{l}\text { King, T. } \\
\text { Aggarwal, N. } \\
\text { Taddeo, M. } \\
\text { Floridi, L. } \\
\text { (2018, United Kingdom) }\end{array}$ & $\begin{array}{l}\text { Sociology } \\
\text { Political science } \\
\text { Ethics }\end{array}$ & $\begin{array}{l}\text { Transversal } \\
\text { Mixed } \\
\text { Non-experimental }\end{array}$ & $\begin{array}{l}\text { Descriptive } \\
\text { Positive effect }\end{array}$ & $\begin{array}{l}\text { Use IA } \\
\text { Data analysis }\end{array}$ & $\begin{array}{l}\text { Crimes against collective security } \\
\text { Crimes against sexual freedom and } \\
\text { indemnity } \\
\text { Crimes against heritage and against } \\
\text { the socio-economic order }\end{array}$ & $\begin{array}{l}\text { Civil use } \\
\text { Crime prevention } \\
\text { Police field }\end{array}$ & $\begin{array}{l}\text { Data management } \\
\text { Decision making } \\
\text { Effective implementation }\end{array}$ \\
\hline $\begin{array}{l}\text { Fiott, D. } \\
\text { Lindstrom, G. } \\
\text { (2018, European Union) }\end{array}$ & $\begin{array}{l}\text { Security } \\
\text { Ethics }\end{array}$ & $\begin{array}{l}\text { Transversal } \\
\text { Qualitative } \\
\text { Non-experimental }\end{array}$ & $\begin{array}{l}\text { Descriptive } \\
\text { Positive effect }\end{array}$ & $\begin{array}{l}\text { Use IA } \\
\text { Data analysis } \\
\text { Multimedia systems }\end{array}$ & Crimes against collective security & $\begin{array}{l}\text { Dual use } \\
\text { Crime mitigation } \\
\text { Police field }\end{array}$ & $\begin{array}{l}\text { Data management } \\
\text { Decision making }\end{array}$ \\
\hline
\end{tabular}




\begin{tabular}{|c|c|c|c|c|c|c|c|}
\hline Authors, year and country & Discipline & Design & Results & Security strategy & Crime typology & Area of application & Limitations \\
\hline $\begin{array}{l}\text { Truntsevsky, Y. V. } \\
\text { Lukiny, I. I. } \\
\text { Sumachev, A. V. } \\
\text { Kopytova, A. V. } \\
\text { (2018, Russia) }\end{array}$ & $\begin{array}{l}\text { Computing } \\
\text { Technology }\end{array}$ & $\begin{array}{l}\text { Longitudinal } \\
\text { Quantitative } \\
\text { Non-experimental }\end{array}$ & $\begin{array}{l}\text { Descriptive } \\
\text { Null effect }\end{array}$ & $\begin{array}{l}\text { Use IA } \\
\text { Data analysis }\end{array}$ & $\begin{array}{l}\text { Incivism/Vandalism } \\
\text { Crimes against heritage and against } \\
\text { the socio-economic order } \\
\text { Crimes of homicide } \\
\text { Crimes of injuries } \\
\text { Other crimes }\end{array}$ & $\begin{array}{l}\text { Civil use } \\
\text { Crime prevention } \\
\text { Police field }\end{array}$ & Not specified \\
\hline $\begin{array}{l}\text { Tahir, Z. } \\
\text { Malek, J. A. } \\
\text { (2018, Malaysia) }\end{array}$ & $\begin{array}{l}\text { Security } \\
\text { Sociology } \\
\text { Political science }\end{array}$ & $\begin{array}{l}\text { Transversal } \\
\text { Mixed } \\
\text { Quasi-experimental }\end{array}$ & $\begin{array}{l}\text { Descriptive } \\
\text { Positive effect }\end{array}$ & $\begin{array}{l}\text { Use conventional strategies } \\
\text { CPTED } \\
\text { Multimedia systems }\end{array}$ & $\begin{array}{l}\text { Incivism/Vandalism } \\
\text { Crimes against heritage and against } \\
\text { the socio-economic order }\end{array}$ & $\begin{array}{l}\text { Civil use } \\
\text { Crime prevention } \\
\text { Police field }\end{array}$ & Not specified \\
\hline $\begin{array}{l}\text { Eastern Alliance for Safe and } \\
\text { Sustainable Transport } \\
\text { (2018, Kyrgyzstan) }\end{array}$ & $\begin{array}{l}\text { Sociology } \\
\text { Political science }\end{array}$ & $\begin{array}{l}\text { Transversal } \\
\text { Mixed } \\
\text { Quasi-experimental }\end{array}$ & $\begin{array}{l}\text { Descriptive } \\
\text { Positive effect }\end{array}$ & $\begin{array}{l}\text { Use conventional strategies } \\
\text { CPTED } \\
\text { Data analysis }\end{array}$ & $\begin{array}{l}\text { Crimes against collective security } \\
\text { Crimes of homicide } \\
\text { Crimes of injuries }\end{array}$ & $\begin{array}{l}\text { Civil use } \\
\text { Crime prevention } \\
\text { Police field }\end{array}$ & Not specified \\
\hline $\begin{array}{l}\text { Schuilenburg, M. } \\
\text { Peeters, R. } \\
\text { (2018, Netherlands) }\end{array}$ & $\begin{array}{l}\text { Sociology } \\
\text { Political science }\end{array}$ & $\begin{array}{l}\text { Longitudinal } \\
\text { Mixed } \\
\text { Quasi-experimental }\end{array}$ & $\begin{array}{l}\text { Descriptive } \\
\text { Positive effect }\end{array}$ & $\begin{array}{l}\text { Use conventional strategies } \\
\text { Data analysis } \\
\text { Multimedia systems }\end{array}$ & $\begin{array}{l}\text { Incivism/Vandalism } \\
\text { Crimes against collective security }\end{array}$ & $\begin{array}{l}\text { Civil use } \\
\text { Crime prevention } \\
\text { Crime mitigation } \\
\text { Police field } \\
\end{array}$ & Not specified \\
\hline $\begin{array}{l}\text { Berry, M. } \\
\text { (2018, United Kingdom) }\end{array}$ & $\begin{array}{l}\text { Sociology } \\
\text { Political science }\end{array}$ & $\begin{array}{l}\text { Longitudinal } \\
\text { Qualitative } \\
\text { Non-experimental }\end{array}$ & $\begin{array}{l}\text { Descriptive } \\
\text { Negative effect }\end{array}$ & $\begin{array}{l}\text { Use conventional strategies } \\
\text { Data analysis }\end{array}$ & Crimes against collective security & $\begin{array}{l}\text { Civil use } \\
\text { Crime prevention } \\
\text { Police field }\end{array}$ & $\begin{array}{l}\text { Data management } \\
\text { Decision making } \\
\text { Effective implementation } \\
\text { Other problems }\end{array}$ \\
\hline $\begin{array}{l}\text { Lee, J. Y. } \\
\text { Kim, K. D. } \\
\text { Kim, K. } \\
\text { (2019, South Korea) }\end{array}$ & $\begin{array}{l}\text { Computing } \\
\text { Technology } \\
\text { Criminology }\end{array}$ & $\begin{array}{l}\text { Transversal } \\
\text { Mixed } \\
\text { Non-experimental }\end{array}$ & $\begin{array}{l}\text { Descriptive } \\
\text { Positive effect }\end{array}$ & $\begin{array}{l}\text { Use conventional strategies } \\
\text { Data analysis } \\
\text { Multimedia systems }\end{array}$ & $\begin{array}{l}\text { Incivism/Vandalism } \\
\text { Crimes against heritage and against } \\
\text { the socio-economic order } \\
\text { Crimes of homicide } \\
\text { Crimes of injuries }\end{array}$ & $\begin{array}{l}\text { Civil use } \\
\text { Crime prevention } \\
\text { Police field }\end{array}$ & Data management \\
\hline $\begin{array}{l}\text { Cho, Y. } \\
\text { Jeong, H. } \\
\text { Choi, A. } \\
\text { Sung, M. } \\
\text { (2019, South Korea) }\end{array}$ & Security & $\begin{array}{l}\text { Transversal } \\
\text { Mixed } \\
\text { Quasi-experimental }\end{array}$ & $\begin{array}{l}\text { Descriptive } \\
\text { Mixed effect }\end{array}$ & $\begin{array}{l}\text { Use conventional strategies } \\
\text { Multimedia systems }\end{array}$ & $\begin{array}{l}\text { Incivism/Vandalism } \\
\text { Crimes against collective security } \\
\text { Crimes against sexual freedom and } \\
\text { indemnity } \\
\text { Crimes against heritage and against } \\
\text { the socio-economic order } \\
\text { Crimes of homicide } \\
\text { Crimes of injuries } \\
\text { Other crimes }\end{array}$ & $\begin{array}{l}\text { Civil use } \\
\text { Crime prevention } \\
\text { Police field }\end{array}$ & $\begin{array}{l}\text { Data management } \\
\text { Other problems }\end{array}$ \\
\hline $\begin{array}{l}\text { Vitunskaite, M. } \\
\text { He, Y. } \\
\text { Brandstetter, T. } \\
\text { Janicke, H. } \\
\text { (2019, United Kingdom) }\end{array}$ & $\begin{array}{l}\text { Computing } \\
\text { Technology } \\
\text { Security }\end{array}$ & $\begin{array}{l}\text { Transversal } \\
\text { Qualitative } \\
\text { Non-experimental }\end{array}$ & $\begin{array}{l}\text { Descriptive } \\
\text { Mixed effect }\end{array}$ & $\begin{array}{l}\text { Use conventional strategies } \\
\text { Data analysis }\end{array}$ & Not specified & $\begin{array}{l}\text { Civil use } \\
\text { Crime prevention } \\
\text { Police field }\end{array}$ & $\begin{array}{l}\text { Data management } \\
\text { Decision making } \\
\text { Effective implementation } \\
\text { Other problems }\end{array}$ \\
\hline $\begin{array}{l}\text { Catlett, C. } \\
\text { Cesario, E. } \\
\text { Talia, D. } \\
\text { Vinci, E. } \\
\text { (2019, United States / Italy) }\end{array}$ & $\begin{array}{l}\text { Computing } \\
\text { Technology }\end{array}$ & $\begin{array}{l}\text { Longitudinal } \\
\text { Mixed } \\
\text { Experimental }\end{array}$ & $\begin{array}{l}\text { Inferential } \\
\text { Positive effect }\end{array}$ & $\begin{array}{l}\text { Use IA } \\
\text { Data analysis }\end{array}$ & $\begin{array}{l}\text { Crimes against heritage and against } \\
\text { the socio-economic order }\end{array}$ & $\begin{array}{l}\text { Civil use } \\
\text { Crime prevention } \\
\text { Police field }\end{array}$ & Not specified \\
\hline $\begin{array}{l}\text { Kitchin, R. } \\
\text { Dodge, M. } \\
\text { (2019, United Kingdom) }\end{array}$ & $\begin{array}{l}\text { Security } \\
\text { Criminology } \\
\text { Sociology } \\
\text { Political science } \\
\end{array}$ & $\begin{array}{l}\text { Transversal } \\
\text { Qualitative } \\
\text { Non-experimental }\end{array}$ & $\begin{array}{l}\text { Descriptive } \\
\text { Mixed effect }\end{array}$ & $\begin{array}{l}\text { Use conventional strategies } \\
\text { Data analysis }\end{array}$ & $\begin{array}{l}\text { Incivism/Vandalism } \\
\text { Crimes against collective security } \\
\text { Crimes against heritage and against } \\
\text { the socio-economic order }\end{array}$ & $\begin{array}{l}\text { Civil use } \\
\text { Crime prevention } \\
\text { Crime mitigation } \\
\text { Police field } \\
\end{array}$ & $\begin{array}{l}\text { Data management } \\
\text { Effective implementation }\end{array}$ \\
\hline
\end{tabular}




\section{g. Quantitative systematic review of literature}

Table 3. Summary of quantitative results

\begin{tabular}{|c|c|c|c|c|}
\hline Category & Variables & Modalities & $\mathrm{F}$ & $\%$ \\
\hline \multirow{8}{*}{ Design } & \multirow{2}{*}{ Temporality of data collection } & Transversal & 14 & 70 \\
\hline & & Longitudinal & 6 & 30 \\
\hline & \multirow{3}{*}{ Approach } & Quantitative & 2 & 10 \\
\hline & & Qualitative & 9 & 45 \\
\hline & & Mixed & 9 & 45 \\
\hline & \multirow{3}{*}{ Research method } & Experimental & 1 & 5 \\
\hline & & Quasi-experimental & 9 & 45 \\
\hline & & Non-experimental & 10 & 50 \\
\hline \multirow{7}{*}{ Results } & \multirow{3}{*}{ Statistics scope } & Descriptive & 18 & 90 \\
\hline & & Inferential & 2 & 10 \\
\hline & & Mixed & 0 & 0 \\
\hline & \multirow{4}{*}{ Effect of the result obtained } & Positive & 12 & 60 \\
\hline & & Negative & 1 & 5 \\
\hline & & Mixed & 6 & 30 \\
\hline & & Null & 1 & 5 \\
\hline \multirow{2}{*}{ Security strategy } & \multirow{2}{*}{ Measures used } & Use Artificial Intelligence & 10 & 50 \\
\hline & & Use conventional strategies & 10 & 50 \\
\hline \multirow{7}{*}{ Crime prevention } & \multirow{7}{*}{ Crime typology } & Incivism/vandalism & 9 & 45 \\
\hline & & Against collective security & 9 & 45 \\
\hline & & Against sexual indemnity & 2 & 10 \\
\hline & & Against heritage & 9 & 45 \\
\hline & & Homicide & 4 & 20 \\
\hline & & Injuries & 4 & 20 \\
\hline & & Other crimes & 2 & 10 \\
\hline \multirow{9}{*}{ Application } & \multirow{3}{*}{ Use } & Civil & 19 & 95 \\
\hline & & Military & 0 & 0 \\
\hline & & Dual & 1 & 5 \\
\hline & \multirow{3}{*}{ Objective } & Investigation & 1 & 5 \\
\hline & & Mitigation & 5 & 25 \\
\hline & & Prevention & 17 & 85 \\
\hline & \multirow{3}{*}{ Field } & Police & 20 & 100 \\
\hline & & Judicial & 0 & 0 \\
\hline & & Both & 0 & 0 \\
\hline \multirow{4}{*}{ Limitations } & \multirow{4}{*}{ Difficulties associated } & Data management & 12 & 60 \\
\hline & & Decision making & 5 & 25 \\
\hline & & Effective implementation & 7 & 35 \\
\hline & & Other problems & 3 & 15 \\
\hline
\end{tabular}

Note: the percentages have been calculated in relation to the total number of articles reviewed $(n=20)$. For this reason, there are variables that can exceed $100 \%$ when different modalities occur at the same time like criminal typologies, the objective of its application and the difficulties associated with the research.

\section{Discussion and conclusions}

Our view at this point is clearly that there is still a lot of work to be done, but some aspects of this review are fairly clear. These include several points. The most common crimes to be prevented are social or property crimes, such as vandalism or theft. These are crimes 
usually committed on the street or in public areas (Cho, Jeong, Choi \& Sung, 2019; Schuilenburg \& Peeters, 2018), so these are areas where the administration in charge of managing public security has more scope to work directly than if these crimes were committed in the private areas (King, Aggarwal, Taddeo \& Floridi, 2019). In addition, given that these are crimes in public settings, it facilitates the administration's development of public policies that include the use of technological systems capable of being installed in the same streets or in public spots (Arikuma \& Mochizuki, 2016; Byun, Nasridinov \& Park, 2014). It opens up a novel way of preventing crime by combining these systems with other public security policies, in accordance with the objectives pursued by public managers. Managers of public safety are called upon to opt in favor of the development of new methods of crime prevention by taking advantage of technology and putting the data obtained to correct use (Zanella \& Vangelista, 2014). These methods are used both to achieve the objective of preventing crime and to provide know-how and information to help public administrations. That is why there are advantages in smart cities in terms of public security which should be seized upon (Maestre, 2015).

At the same time, the use of artificial intelligence in the approaches is increasing, especially in connection with the management of large-scale data (Ghosh, Chun, Shafiq \& Adam, 2016). Artificial intelligence presents benefits that should be taken advantage of, since it facilitates and simplifies processes that can be complex if accomplished manually (Truntsevsky, Lukiny, Sumachev \& Kopytova, 2018). The automation allows data to be generated constantly and to obtain results in real time (Bartoli et al., 2013). Consequently, its implementation will be particularly useful when dealing or managing large amounts of data and conducting suitable analyses. 
Furthermore, an ethical applicability of artificial intelligence may be challenged by posing some dilemmas depending on the circumstances. As commented above, there are concepts such as transparency, confidentiality, citizen privacy, etc., to be considered and limits to be established that cannot be exceeded (Macmanus, Caruson \& Mcphee, 2013; Kitchin \& Dodge, 2019). It is also necessary to explain to citizens these concepts and offer the possibility to complain if the measures used violate their rights in some way (Elmaghraby \& Losavio, 2014). In summary, the implementation of security systems in smart cities implies a profound ethical reflection in which different stakeholders participate in the establishment of an appropriate framework for the display of the solutions (Prislan \& Slak, 2018).

Considering the aforementioned issues, it becomes clear that research in this field would have to be followed up and further steps would have to be considered to enhance security in smart cities, by exploiting the potential they offer. For comprehensive and realistic studies on this subject, it is of the utmost importance that managers of public security administrations trust in the figure of the expert in criminology who, in collaboration with a multidisciplinary panel, will be able to offer a sound picture of the behaviors to be prevented, and how to interpret the results generated.

\section{References}

Alawadhi, S., Aldama-Nalda, A., Chourabi, H., Gil-Garcia, J. R., Leung, S., Mellouli, S., Nam, T., Pardo, T., Scholl, H. \& Walker, S. (2012). Building understanding of smart city initiatives. International conference on electronic government, 40-53. doi:10.1007/978-3-642-33489-4_4 
Arikuma, T., \& Mochizuki, Y. (2016). Intelligent multimedia surveillance system for safer cities. APSIPA Transactions on Signal and Information Processing, 5. doi:10.1017/atsip.2016.7

Bartoli, G., Fantacci, R., Gei, F., Marabissi, D., \& Micciullo, L. (2013). A novel emergency management platform for smart public safety. International Journal of Communication Systems, 28(5), 928-943. doi:10.1002/dac.2716

Berry, M. (2018). Technology and organised crime in the smart city: an ethnographic study of the illicit drug trade. City, Territory and Architecture, 5(1), 1-11. doi:10.1186/s40410-018-0091-7

Byun, J. Y., Nasridinov, A., \& Park, Y. H. (2014). Internet of things for smart crime detection. Contemporary Engineering Sciences, 7(15), 749-754. doi:10.12988/ces.2014.4685

Carreño, P., Gutierrez, F. J., Ochoa, S. F., \& Fortino, G. (2015). Supporting personal security using participatory sensing. Concurrency and Computation: Practice and Experience, 27(10), 2531-2546. doi:10.1002/cpe.3450

Catlett, C., Cesario, E., Talia, D., \& Vinci, A. (2019). Spatio-temporal crime predictions in smart cities: A data-driven approach and experiments. Pervasive and Mobile Computing, 53, 62-74. doi:10.1016/j.pmcj.2019.01.003

Chiodi, S. I. (2016). Crime prevention through urban design and planning in the smart city era. Journal of Place Management and Development 9(2), 137-152. doi:10.1108/jpmd-09-2015-0037

Cho, Y., Jeong, H., Choi, A., \& Sung, M. (2019). Design of a connected security lighting system for pedestrian safety in smart cities. Sustainability, 11(5), 1308. doi:10.3390/su11051308 
Colado, S., Gutierrez, A., Vives, C. \& Valencia, E. (2014). Smart city. Hacia la gestión inteligente. Marcombo Ediciones técnicas.

Deloitte. (2015). Smart Cities. How rapid advances in technology are reshaping our economy and society. Available in: https://www2.deloitte.com/content/dam/Deloitte/tr/Documents/publicsector/deloitte-nl-ps-smart-cities-report.pdf

Eastern Alliance for Safe and Sustainable Transport. (2018). Pedestrian safety in Bishkek's smart cities concept.

Elmaghraby, A. S., \& Losavio, M. M. (2014). Cyber security challenges in Smart Cities: Safety, security and privacy. Journal of advanced research, 5(4), 491-497. doi:10.1016/j.jare.2014.02.006

Eremia, M., Toma, L., \& Sanduleac, M. (2017). The smart city concept in the $21 \mathrm{st}$ century. Procedia Engineering, 181, 12-19. doi:10.1016/j.proeng.2017.02.357

Fiott, D., \& Lindstrom, G. (2018). Artificial Intelligence: What implications for EU security and defence. European Institute for Security Studies (EUISS), Brief SSUE 10.

Ghosh, D., Chun, S., Shafiq, B., \& Adam, N. (2016). Big data-based smart city platform: Real-time crime analysis. Proceedings of the 17th International Digital Government Research Conference on Digital Government Research, 58-66. doi:10.1145/2912160.2912205

Griffith University, School of Environment and Science. Brisbane, Australia: Systematic quantitative literature review. Available in https://www.griffith.edu.au/griffithsciences/school-environment-science/research/systematic-quantitative-literaturereview 
Huesa, J. (07/08/2019). Smart City y seguridad ciudadana: la inteligencia al servicio de eventos multitudinarios (ejemplo: Semana Santa de Sevilla). eSmartCity. Available in https://www.esmartcity.es/comunicaciones/comunicacion-smartcity-seguridad-ciudadana-inteligencia-servicio-eventos-multitudinarios-ejemplosemana-santa-sevilla

Khatoun, R., \& Zeadally, S. (2016). Smart cities: Concepts, architectures, research opportunities. Communications of the ACM, 59(8), 46-57. doi:10.1145/2858789

King, T. C., Aggarwal, N., Taddeo, M., \& Floridi, L. (2019). Artificial intelligence crime: An interdisciplinary analysis of foreseeable threats and solutions. Science and engineering ethics, 1-32. doi:10.1007/s11948-018-00081-0

Kitchin, R., \& Dodge, M. (2019). The (in) security of smart cities: vulnerabilities, risks, mitigation, and prevention. Journal of Urban Technology, 26(2), 47-65. doi:10.1080/10630732.2017.1408002

Kumar, R. (2019). Street light monitoring using smartphones. International Journal of Engineering and Advanced Technology (IJEAT) 9(1), 2249-8958.

Kummitha, R. (2019). Smart cities and entrepreneurship: An agenda for future research. Technological Forecasting and Social Change, 149. doi:10.1016/j.techfore.2019.119763

Lee, J. Y., Kim, K. D., \& Kim, K. (2019). A study on improving the location of CCTV cameras for crime prevention through an analysis of population movement patterns using mobile big data. KSCE Journal of Civil Engineering, 23(1), 376387. doi:10.1007/s12205-018-1486-4

Lin, Y. L., Chen, T. Y., \& Yu, L. C. (2017). Using machine learning to assist crime prevention. In 2017 6th IIAI International Congress on Advanced Applied Informatics (IIAI-AAI), 1029-1030. doi:10.1109/iiai-aai.2017.46 
Lodato, T., French, E., \& Clark, J. (2018). Open government data in the smart city: Interoperability, urban knowledge, and linking legacy systems. Journal of Urban Affairs, 1-15. doi:10.1080/07352166.2018.1511798

Macmanus, S. A., Caruson, K., \& Mcphee, B. D. (2013). Cybersecurity at the Local Government Level: Balancing Demands for Transparency and Privacy Rights. Journal of Urban Affairs, 35(4), 451-470. doi:10.1111/j.1467-9906.2012.00640.x

Maestre, G. (2015). Revisión de literatura sobre ciudades inteligentes: una perspectiva centrada en las TIC. Ingeniare, (19), 137-149. doi:10.18041/19092458/ingeniare.19.531

Moher, D., Liberati, A., Tetzlaff, J., \& Altman, D. G. (2009). Preferred reporting items for systematic reviews and meta-analyses: the PRISMA statement. Annals of internal medicine, 151(4), 264-269.

Mueller, T. (31/08/2017). Redefining the smart city concept: A new smart city definition. Bee Smart City. Available in https://hub.beesmart.city/strategy/en/towards-anew-smart-city-definition

Perrot, P. (2017). What about AI in criminal intelligence? From predictive policing to AI perspectives. European Law Enforcement Research Bulletin, (16), 65-75.

Pickering, C., \& Byrne, J. (2014). The benefits of publishing systematic quantitative literature reviews for $\mathrm{PhD}$ candidates and other early-career researchers. Higher Education Research \& Development, 33(3), 534-548. doi:10.1080/07294360.2013.841651

Prislan, K., \& Slak, B. (2018). Analysis of the Relationship Between Smart Cities, Policing and Criminal Investigation. Journal of Criminal Justice and Security, (4), $389-413$.

Rivero, M. (2017). Smart Cities: Una visión para el ciudadano. LID Editorial. 
Schuilenburg, M., \& Peeters, R. (2018). Smart cities and the architecture of security: pastoral power and the scripted design of public space. City, Territory and Architecture, 5(1), 13. doi:10.1186/s40410-018-0090-8

Sormani, R., Soldatos, J., Vassilaras, S., Kioumourtzis, G., Leventakis, G., Giordani, I., \& Tisato, F. (2016). A serious game empowering the prediction of potential terrorist actions. Journal of Policing, Intelligence and Counter Terrorism, 11(1), 30-48. doi:10.1080/18335330.2016.1161222

Tahir, Z., \& Malek, J. A. (2018). Elements of security for a gated and guarded community in the context of smart living. e-Bangi, 14(3).

Townsend, A. (2013). Smart Cities. Big data, civic hackers and the quest for a new utopia. W. W. Norton \& Company, Inc.

Truntsevsky, Y., Lukiny, I., Sumachev, A., \& Kopytova, A. (2018). A smart city is a safe city: the current status of street crime and its victim prevention using a digital application. MATEC Web of Conferences 170, 01067. doi:10.1051/matecconf/201817001067

Vattapparamban, E., Güvenç, İ., Yurekli, A. İ., Akkaya, K., \& Uluağaç, S. (2016). Drones for smart cities: Issues in cybersecurity, privacy, and public safety. 2016 International Wireless Communications and Mobile Computing Conference (IWCMC), 216-221. doi:10.1109/iwcmc.2016.7577060

Vidal-Tejedor, N. (2015). La Smart City. Las ciudades inteligentes del futuro. Editorial UOC.

Vitunskaite, M., He, Y., Brandstetter, T., \& Janicke, H. (2019). Smart cities and cyber security: Are we there yet? A comparative study on the role of standards, third party risk management and security ownership. Computers \& Security, 83, 313331. doi:10.1016/j.cose.2019.02.009 
Zanella, A. \& Vangelista, L. (2014). Internet of things for smart cities. IEEE Internet of Things journal, 1(1), 22-32. doi:10.1109/jiot.2014.2306328 